\title{
Rhino-orbito-cerebral mucormycosis complicated with an ophthalmic artery occlusion followed by subarachnoid hemorrhage
}

\author{
Kun Wook Kang, Young Ki Kwon, Jae Pil Shin, In Taek Kim, Dong Ho Park \\ Department of Ophthalmology, Graduate School of Medicine, Kyungpook National University, Daegu, South Korea; \\ *Corresponding Author: sarasate2222@gmail.com
}

Received 14 July 2013; revised 5 August 2013; accepted 12 August 2013

Copyright (C) 2013 Kun Wook Kang et al. This is an open access article distributed under the Creative Commons Attribution License, which permits unrestricted use, distribution, and reproduction in any medium, provided the original work is properly cited.

\begin{abstract}
A 70-year-old female with poorly controlled diabetes developed sudden visual loss, ptosis and complete ophthalmoplegia of the right eye. Funduscopic examination showed the pale retina and the cherry red spot in the right eye. Fluorescein angiography and indocyanine green angiography demonstrated the absence of retinal arterial filling and choroidal perfusion in the right eye even 20 minutes after injecting the dye. The patient was diagnosed with right ophthalmic artery occlusion. Computed tomography (CT) showed diffuse mucosal thickening in the right ethmoidal sinus. Based on the clinical findings and endoscopic biopsy result, mucormycosis was confirmed. Amphotericin B (40 mg/day) and ceftriaxone ( $2 \mathrm{~g} /$ day) were intravenously administered. Despite the improvement of the right ethmoidal sinusitis and the right proptosis, the patient deteriorated into a comatose state after 19 days of systemic amphotericin B therapy. Although the previous CT showed no cerebral aneurysm, a repeated CT showed newly developed posterior communicating artery aneurysm and the subarachnoid hemorrhage. Despite the amphotericin B treatment and the improvement of the sinusitis, mucormycosis could cause sudden cerebral aneurysm rupture and subarachnoid hemorrhage resulting in coma.
\end{abstract}

Keywords: Intracranial Aneurysm; Mucormycosis; Ophthalmic Artery; Rhino-Orbito-Cerebral Mucormycosis; Subarachnoid Hemorrhage

\section{INTRODUCTION}

Mucormycosis is a fungal infection caused by the family Mucoraceae-pathogenic genera Rhizopus, Absidia and Mucor [1]. Infections typically occur in immunocompromised patients with diabetic ketoacidosis, lymphoma, leukemia, corticosteroid treatment, radiation therapy, chemotherapy, myelodysplastic syndrome, aplastic anemia and the acquired immunodeficiency syndrome [2]. Rhino-orbito-cerebral mucormycosis is a rare opportunistic fungal infection that is potentially fatal.

We report a rare case of rhino-orbito-cerebral mucormycosis complicated with an ophthalmic artery occlusion followed by sudden onset subarachnoid hemorrhage.

\section{CASE REPORT}

A 70-year-old female presented to hospital with a three day history of visual loss and ptosis of the right eye. The patient was diagnosed with diabetes 30 years ago but took the medication irregularly for recent 2 weeks. Blood glucose level at admission was $516 \mathrm{mg} / \mathrm{dL}$.

The visual acuity was no light perception in the right eye and 20/40 in the left eye with a relative afferent pupillary defect on the right side. Complete restriction of ocular motility in all fields of gaze, ptosis and proptosis of the right eye were observed. Funduscopic examination showed the pale, edematous retina and the cherry red spot in the right eye (Figure 1(A)). Fluorescein angiography and indocyanine green angiography demonstrated the absence of retinal arterial filling and choroidal perfusion in the right eye even 20 minutes after injecting the dye (Figure 1(B)). The patient was diagnosed with right ophthalmic artery occlusion.

Computed tomography (CT) of the brain and orbit showed diffuse mucosal thickening in the right ethmoidal sinus but there was no involvement of the cavernous sinus or the cavernous carotid artery (Figure 1(C)). The patient underwent an urgent endoscopic nasal exploration. Rhinologic exam revealed a dark brown colored eschar 
at the right nasal septum. The pathologic examination of the surgical specimen at the right nasal septum revealed broad, aseptate hyphae, morphologically consistent with mucormycosis. The definitive diagnosis of mucormycosis was made on histopathologic examination (Figure 1(D)). Amphotericin B (40 mg/day) and ceftriaxone (2 g/day) were intravenously administered.

Despite the improvement of the right ethmoidal sinusitis and the right proptosis after 9 days of systemic amphotericin B therapy (Figure 2(A)), the patient deteriorated into a comatose state after 19 days of systemic amphotericin B therapy. Although the previous CT showed no cerebral aneurysm (Figure 2(C)), a repeated CT scan of the brain showed newly developed right posterior communicating artery aneurysm and the subarachnoid hemorrhage with a right temporal lobe hematoma (Figures 2(B) and 2(D)).

Rhino-orbito-cerebral mucormycosis was diagnosed. Endovascular coil embolization of posterior communicating artery and craniotomy for subarachnoid hemorrhage were performed. The patient was still comatose.

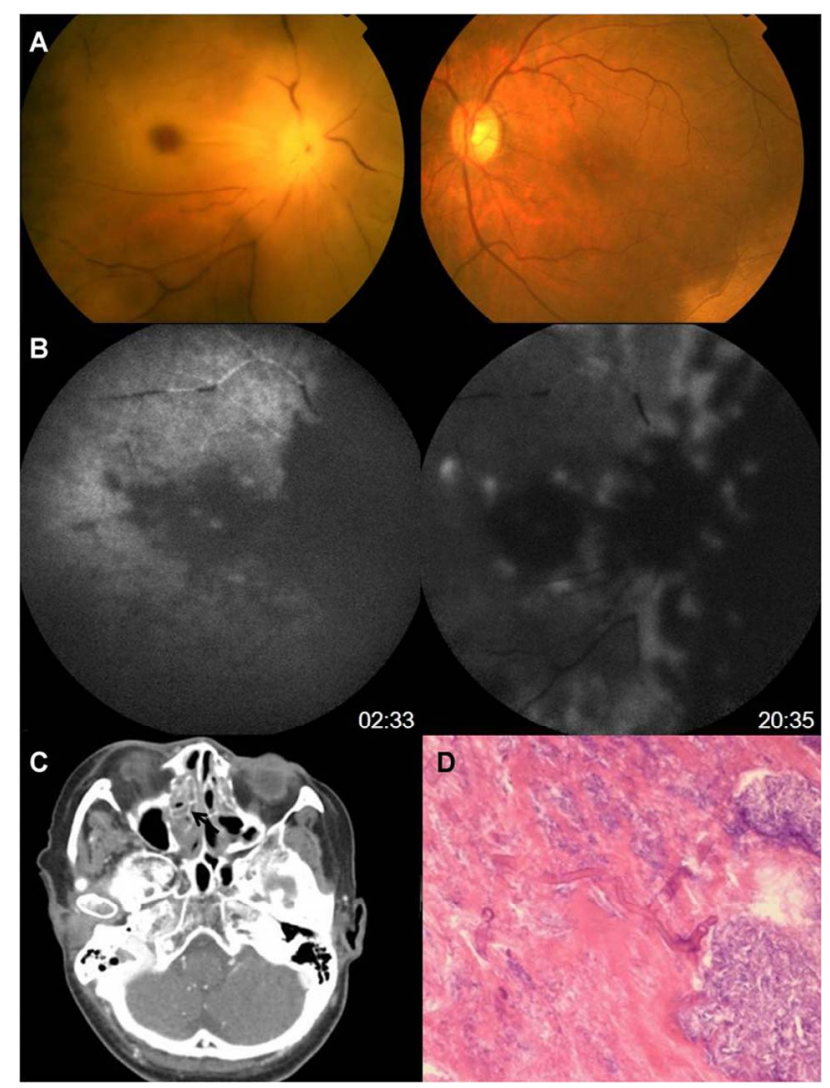

Figure 1. Fundus photograph revealed a pale retina and cherry red spot in the right eye (A). Fluorescein angiography revealed absence of retinal arterial filling and choroidal perfusion in the right eye (B). Computed tomography showed diffuse mucosal thickening in the right ethmoidal sinus (C, black arrow). Biopsy of right nasal septum showed broad aseptate fungal hyphae with acute angle branching (H\&E, ×400) (D).

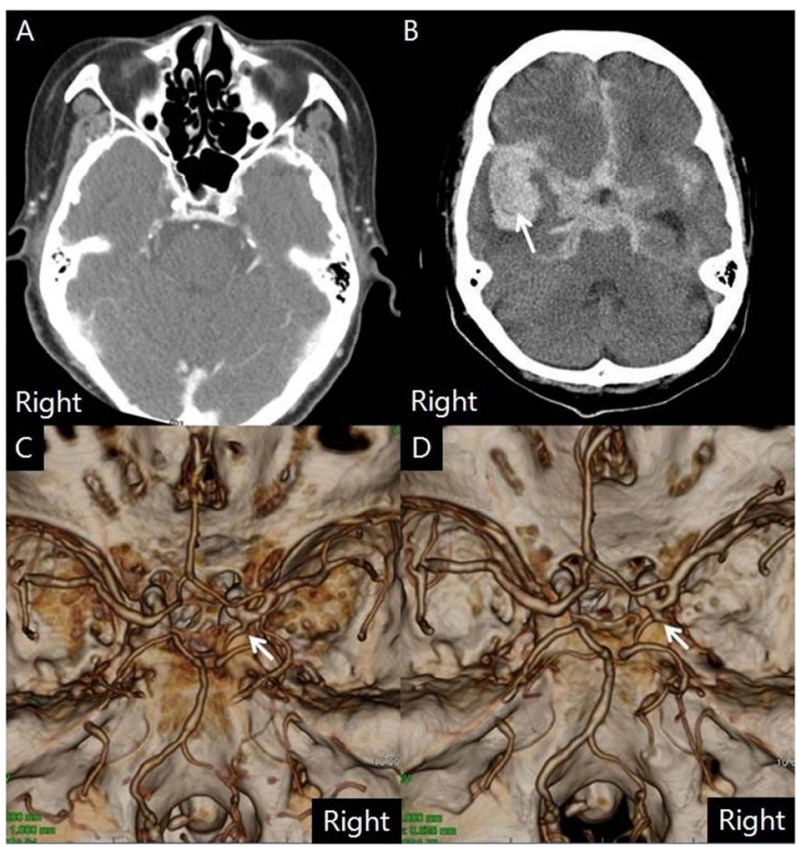

Figure 2. Brain CT showing the improvement of the right ethmoidal sinusitis (A). Brain CT showing the subarachnoid hemorrhage with a right temporal lobe hematoma ((B), arrow). Although the previous CT showed no cerebral aneurysm ((C), arrow), a repeated CT scan showed newly developed right posterior communicating artery aneurysm ((D), arrow).

\section{DISCUSSION}

Rhino-orbito-cerebral mucormycosis is a fulminant opportunistic infection in diabetic or immunocompromised patients. In the present case, the patient had poorly controlled diabetes and the fungal ethmoidal sinusitis. The patient was diagnosed with right ophthalmic artery occlusion and complete ophthalmoplegia. Visual loss results from retinal infarction due to hypoperfusion of the central retinal artery, anterior ischemic optic neuropathy and choroidal ischemia due to compromised posterior ciliary arteries [3]. It is caused by mucorthrombosis of the ophthalmic artery and direct invasion of the optic nerve by fungus infection [4]. Ophthalmoplegia results from impaired blood supply to the extraocular muscles or to the ocular motor nerves [5].

Direct local invasion and spread via blood stream are two common modes of spreading in cephalic mucormycosis. By spreading through vessels and nerves, this fungal infection causes the vascular invasion, cranial nerve involvement, distant brain abscess, septic (mycotic) cerebral embolism, cerebral infarction and aneurysm [6,7].

Infectious aneurysms of cerebral blood vessels are rare, representing only $2 \%$ to $5 \%$ of all intracranial aneurysms. Although infectious aneurysms usually result from bacterial infection, they may also result from fungal infection, causing true mycotic or fungal aneurysms. Fungal intracranial aneurysms arise almost exclusively in the 
setting of an extravascular infection in an immunocompromised host [8]. In the present case, the patient with poorly controlled diabetes complained of only ocular symptom and brain CT showed no infectious cerebral aneurysm at admission. After 9 days of systemic amphotericin B therapy, brain CT showed the improvement of the proptosis of the right eye and the resolution of mucosal thickening of ethmoidal sinus. However, 19 days after systemic amphotericin B therapy, the patient deteriorated into a comatose state. Brain CT showed the diffuse subarachnoid hemorrhage with a right temporal lobe hematoma due to ruptured aneurysm. Within 19 days, new cerebral aneurysm developed and progressed rapidly. It supported our suspicion of a fungal aneurysm.

Survival of mucormycosis with intracerebral involvement was thought to be virtually impossible until the first case of clinical recovery reported in 1955 [9]. The introduction of amphotericin B in the 1960s reduced mortality from $70 \%$ to $40 \%$ [10]. Nevertheless, the prognosis of rhino-orbito-cerebral mucormycosis remains poor and 20 to $50 \%$ of patients expire [11]. In the present case, the ethmoidal sinusitis and the proptosis of the right eye were improved after amphotericin B treatment. However the subarachnoid hemorrhage due to ruptured aneurysm occurred in a short period. Although endovascular coil embolization of posterior communicating artery and craniotomy for subarachnoid hemorrhage were performed, the patient was still comatose.

In conclusion, systemic amphotericin B antifungal treatment improves the sinusitis and the proptosis. Nevertheless, new cerebral aneurysm can develop in a short period and it can cause subarachnoid hemorrhage. Ophthalmologists should raise the suspicion of the infectious mycotic cerebral aneurysm and the subarachnoid hemorrhage resulting in coma, even if the sinusitis is improved after amphotericin B treatment.

\section{ACKNOWLEDGEMENTS}

This research was supported by the Basic Science Research Program through the National Research Foundation of Korea (NRF) funded by the Ministry of Education, Science and Technology (2012004585) and by the Korea Health Technology R\&D Project, Ministry of Health \& Welfare, Republic of Korea (A111345).

\section{REFERENCES}

[1] Koc, Z., Koc, F., Yerdelen, D. and Ozdogu, H. (2007) Rhino-orbital-cerebral mucormycosis with different cerebral involvements: Infarct, hemorrhage, and ophthalmoplegia. International Journal of Neuroscience, 117, 1677 1690. doi:10.1080/00207450601050238

[2] Haliloglu, N.U., Yesilirmak, Z., Erden, A. and Erden, I. (2008) Rhino-orbito-cerebral mucormycosis: Report of two cases and review of the literature. Dentomaxillofacial Radiology, 37, 161-166. doi:10.1259/dmfr/14698002

[3] Yang, S.W., Kim, S.Y., Chung, J. and Kim, K.B. (2000) Two cases of orbital infarction syndrome. Korean Journal of Ophthalmology, 14, 107-111.

[4] Hussain, S., Salahuddin, N., Ahmad, I., Salahuddin, I. and Jooma, R. (1995) Rhinocerebral invasive mycosis: Occurrence in immunocompetent individuals. European Journal of Radiology, 20, 151-155. doi:10.1016/0720-048X(95)00644-6

[5] Zimmerman, C.F., Van Patten, P.D., Golnik, K.C., Kopitnik Jr., T.A. and Anand, R. (1995) Orbital infarction syndrome after surgery for intracranial aneurysms. Ophthalmology, 102, 594-598.

[6] Chimelli, L. and Mahler-Araujo, M.B. (1997) Fungal infections. Brain Pathology, 7, 613-627. doi:10.1111/j.1750-3639.1997.tb01078.x

[7] Peterus, T., Teguh, T. and Daofu, D. (2004) Fatal strokes in patients with rhino-orbito-cerebral mucormycosis and associated vasculopathy. Scandinavian Journal of Infectious Diseases, 36, 643-648. doi:10.1080/00365540410020794

[8] Asari, S., Nishimoto, A. and Murakami, M. (1988) A rare case of cerebral aspergillus aneurysm at the site of temporary clip application. No Shinkei Geka, 16, 1079-1082.

[9] Harris, J.S. (1955) Mucormycosis; report of a case. Pediatrics, 16, 857-867.

[10] Shah, P.D., Peters, K.R. and Reuman, P.D. (1997) Recovery from rhinocerebral mucormycosis with carotid artery occlusion: A pediatric case and review of the literature Pediatric Infectious Disease Journal, 16, 68-71. doi:10.1097/00006454-199701000-00015

[11] Toumi, A., Larbi Ammari, F., Loussaief, C., Hadhri, R., Ben Brahim, H., Harrathi, K., Ben Romdhane, F., Koubaa, J. and Chakroun, M. (2012) Rhino-orbito-cerebral mucormycosis: Five cases. Médecine et Maladies Infectieuses, 42, 591-598. doi:10.1016/j.medmal.2012.10.001 